\title{
Hirschsprung's Disease with Bowel Duplication A Rarest of Rare Anomalous Association
}

\begin{abstract}
Hirschsprung's Disease is an uncommon cause of functional intestinal obstruction in neonates, especially females. Duplication of bowel is also a rare cause of mechanical obstruction in children at birth. An association of these two congenital anomalies is not reported in the available English language literature till date. ${ }^{1,2}$ We hereby present a female neonate with these two anomalies successfully managed at our center.
\end{abstract}

Keywords: hirschsprung's disease, duplication cysts, neonatal intestinal obstruction, bowel anomalies
Volume 7 Issue 2 - 2017

\author{
Shera AH,' Shera TA, ${ }^{2}$ Shera FA, ${ }^{2}$ Sanduqji \\ $\mathrm{H},{ }^{\prime}$ Hassan $\mathrm{F}^{\prime}$ \\ 'Department of Pediatric Surgery, Maternity and Children's \\ Hospital Madina, India \\ ${ }^{2}$ University of Sher I Kashmir Institute of Medical Sciences, India
}

Correspondence: Altaf Hussain Shera, M.S, M.Ch, FICS,

Professor and Head Department of Pediatric surgery, Sher-i-

Kashmir Institute of Medical Sciences, Srinagar, J\&K, India,

Email altafhussainshera@gmail.com

Received: August 22, 2017 | Published: October 05, 2017

\section{Case report}

A full-term female neonate, LBW $2.38 \mathrm{~kg}$, product of normal spontaneous vaginal delivery, having an Apgar score 9 at 1 min and 10 at $5 \mathrm{~min}$, presented with greenish vomiting and failure to pass meconium for more than 48 hours after birth. The infant developed progressive abdominal distention while under observation in the neonatal ICU. On examination, the infant looked generally well, maintaining on room air, vital signs were normal and no dismorphic features. Abdomen was distended all over, there was no visible peristalsis or visible bowel loops with normal overlying skin. It was soft and lax, with no palpable mass, but had exaggerated bowel sounds. Digital rectal examination revealed a normal anal orifice with normal muscle tone. Baby passed little sticky meconium after rectal stimulation, but no gush of gas or stool on withdrawal of finger. A preliminary clinical impression of meconium plug syndrome was made, and conservative treatment started including fleet enema, but unfortunately, the condition of the patient did not improve in spite of repeated enemas. Blood counts, Hemoglobin, serum chemistry, renal functions were within normal values. Liver function showed unconjugated hyperbilirubinemia, but other parameters were normal. Plain abdominal $\mathrm{x}$ ray revealed dilated bowel loops all over the abdomen, no air fluid levels, no free intraperitoneal gas, no intramural gas, and no evidence of any mass lesion. However it revealed diffuse gaseous distention of colon, more prominent on the left side. Gastrograffin contrast enema study was undertaken, for diagnostic as well as therapeutic purposes, due to suspicion of meconium plug syndrome. It revealed classic cone shaped narrowing in recto sigmoid area, with proximal dilatation of bowel and distal narrowing suggesting the presence of Hirschsprung's disease (Figure 1).

The dye was retained in the bowel for more than 24 hours. Baby kept NPO and was put on TPN, frequent OGT aspiration. Baby was monitored continuously and prepared for surgery. Repeated $x$ ray of the abdomen revealed an increase in bowel distention and appearance of air fluid levels. Exploratory laparotomy was performed on day six of life. Intraoperatively, we found dilated large colon, with transitional zone at rectosigmoid level, and distal collapsed bowel (Figure 2). Multiple full thickness biopsies were taken from the collapsed, dilated bowel loops as well as the transitional zone. The exploration of the rest of the bowel revealed the presence of a moderate sized mesenteric cyst in the mid ileum (Figure 3 ). The cyst was quite tense, thick walled, stretching the adjoining loop of ilium and causing partial intestinal obstruction. In view of these finding, a wedge resection of the cystic lesion along with the affected bowel loop was undertaken, and end to end anastomosis of the ileum was performed. A divided colostomy was performed just above the transition zone and the abdomen closed.

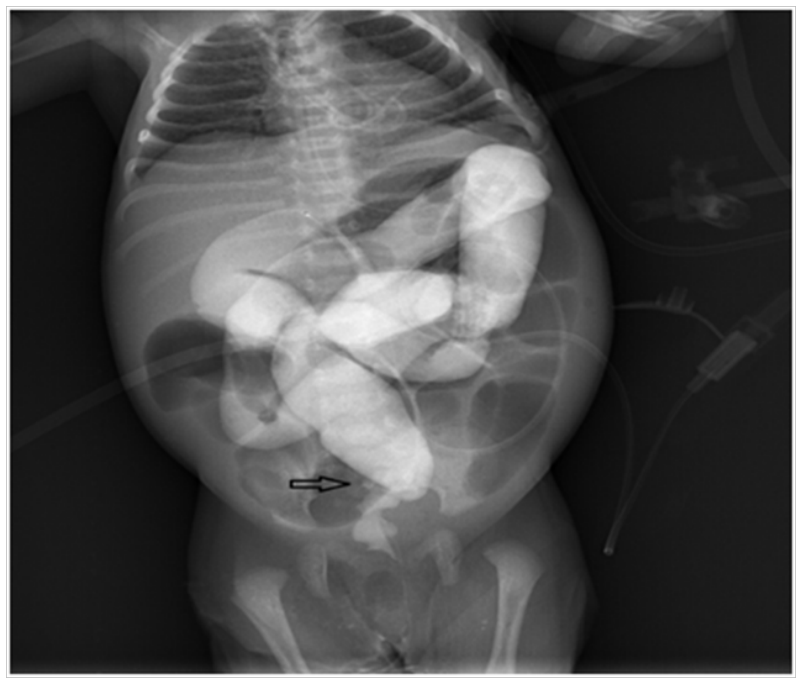

Figure I Proximal dilatation of bowel.

Post operatively, the baby recovered smoothly. The colostomy started functioning on first post-operative day and abdominal wound healed in a week. However, on the fifth post-operative day, the baby developed supra ventricular tachycardia (SVT-AVRT) (Figure 4), and was stabilized by administration of adenosine and propranolol in consultation with pediatric cardiologist. The baby is suspected to have Wolff Parkinson White syndrome (WPW) and is presently under evaluation by the pediatric cardiology team. Histopathological examination revealed the presence of normal ganglion cells in the dilated segment, sparse ganglion cells in the transitional zone and absence of ganglion cells in the distal collapsed bowel, confirming the diagnosis of Hirschsprung's disease. ${ }^{1,2}$ The cystic lesion in the ileum exhibited the features of a classical duplication cyst on HPE. The resected segment of ilium had normal ganglion cells. The patient 
discharged a week after surgery and waiting for definitive surgical procedure for Hirschsprung's disease at a later date.

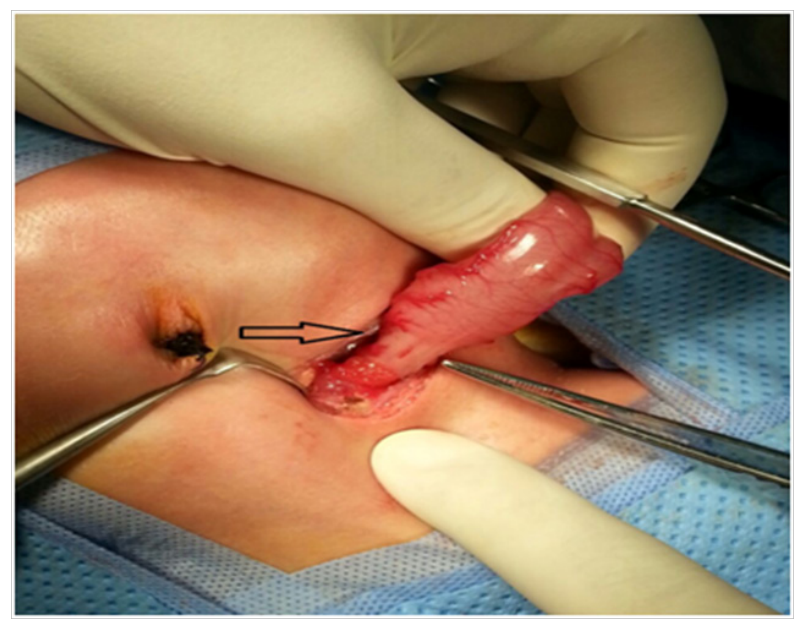

Figure 2 Distal collapsed bowel.

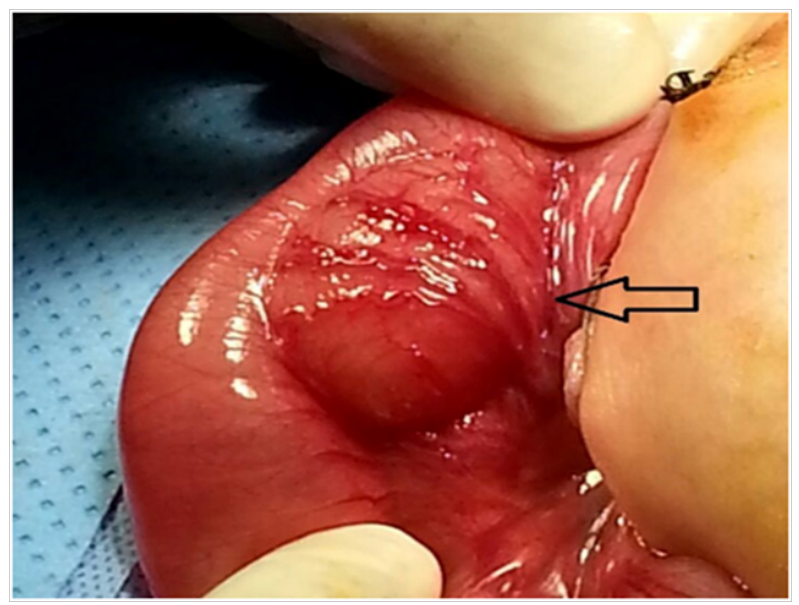

Figure 3 Moderate sized mesenteric cyst.

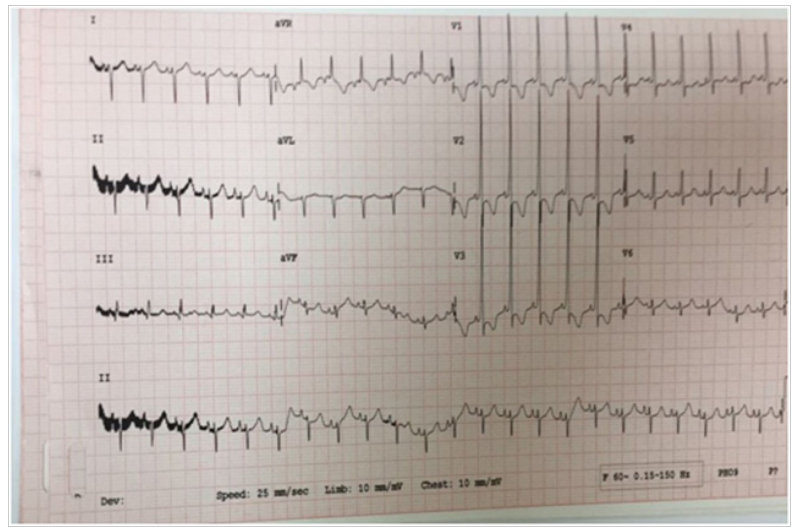

Figure 4 Baby developed supra ventricular tachycardia (SVT-AVRT).

\section{Discussion}

Hirschsprung's disease usually presents as intestinal obstruction in the neonatal period, with the incidence 1 in 5000 live births, having a male to female ratio of $4: 1 .^{3}$ Associated anomalies are present in $22 \%$ of cases. The usual associations are Down's syndrome, defects in cardiac septations, tetralogy of Fallot, and Dandy-walker syndrome.
Duplication of intestine is a more rare disease entity presenting at birth. Ileum is the most common site of occurrence $(60 \%) .{ }^{1}$ Other organs like esophagus, duodenum, stomach and colon are involved in only $4 \%-18 \%$ of all cases. ${ }^{4,5}$ The term intestinal duplication cyst was used, for the first time by Fitz and its further classification was described by Gross in 1950. Duplication cyst may present in any part of GI tract, from esophagus to rectum, however the commonest site is the small bowel and $60 \%$ are found in relation to ileum. Approximately $80 \%$ lesions are cystic, and the remaining tubular. ${ }^{5}$ Tubular lesions may be double barrel type (80\%) or Y shaped (20\%). Cystic lesions are usually closed type, whereas tubular are usually communicating. Duplications are usually located on the mesenteric side of the bowel, sharing a common muscular wall and blood supply. Usually they are asymptomatic and only $30 \%$ produce symptoms at birth. However, $75 \%$ of patients present in first 2 years of life and the remaining present in subsequent years. The usual symptoms are abdominal pain, bleeding or intestinal obstruction. The recommended treatment, even in asymptomatic cases, is surgical resection of the lesion along with involved intestine due to possibility of complications like perforation, obstruction, bleeding and rarely malignancy. An association of duplication cyst and Hirschsprung's disease is hitherto not reported in the available English medical literature to the best of our knowledge. We wished to share this experience with an idea to enhance the awareness regarding possibility of existence of this extremely rare association of congenital anomalies. A possible explanation for the coexistence of these two conditions would be an ischemic insult occurring during embryogenesis and interfering both with the gut canalization and neural crest migration. ${ }^{6,7}$

\section{Conclusion}

A timely diagnosis and appropriate therapeutic intervention is necessary to reduce the morbidity and reduce mortality in such complex congenital malformations. To achieve this goal awareness on the part of treating clinicians and a high index of suspicion about the possibility such unusual and hitherto unknown combination of lesions is necessary especially in the neonates.

\section{Acknowledgments}

None.

\section{Conflict of interest}

The athors declare

\section{Source of dupport}

Nil.

\section{References}

1. Grosfeld JL, O'neill JA, Fonkalsurd EW, et al. Pediatric surgery (7th edn), Elsevier, 1 Chapter 2012. 101:pp. 265-278.

2. Holcomb GW, Murphy PJ, Ostlie DJ. Ahcraft's Pediatric surgery (6th edn), Elsevier Saunders, Chapter 34. 2014. pp. 474-491.

3. Suita S, Taguchi T, Ieiri S, et al. Hirschsprung's disease in Japan:analysis of 3852 patients based on a nationwide survey in 30 years. $J$ Pediatr Surg. 2005;40(1):197-201

4. Puligandla PS, Nguyen LT, St-VilD, et al. Gastrointestinal duplications. Journal of Pediatr Surgery. 2003;38(5):740-744.

5. Holcomb GW, Gheissari A, O'Neill JA, et al. Surgical management of alimentary tract duplications. Ann Surg. 1989;209(2):167-174. 
6. Taguchi T, Suita S, Hirata Y, et al. Abnormally shaped arteries in the intestine of children with Hirschsprung's disease:etiological considerations relating to ischemic theory. $J$ pediatric gastroenterol nutr. 1994;18(2):200-204.
7. Macpherson RI. Gastrointestinal tract duplications:clinical, pathologic, etiologic, and radiologic considerations. Radiographics. 1993;13(5):1063-1080. 